\title{
Integrated Planning of Electric Power Distribution Networks
}

\author{
A. M. Cossi, J. R. S. Mantovani, Member IEEE
}

\begin{abstract}
In this work, a heuristic model for integrated planning of primary distribution network and secondary distribution circuits is proposed. A Tabu Search (TS) algorithm is employed to solve the planning of primary distribution networks. Evolutionary Algorithms (EA) are used to solve the planning model of secondary networks. The planning integration of both networks is carried out by means a constructive heuristic taking into account a set of integration alternatives between these networks. These integration alternatives are treated in a hierarchical way. The planning of primary networks and secondary distribution circuits is carried out based on assessment of the effects of the alternative solutions in the expansion costs of both networks simultaneously. In order to evaluate this methodology, tests were performed for a real-life distribution system taking into account the primary and secondary networks.
\end{abstract}

Keywords - Secondary circuits, power flow, evolutionary algorithms, electric power distribution system.

\section{NOMENCLATURA}

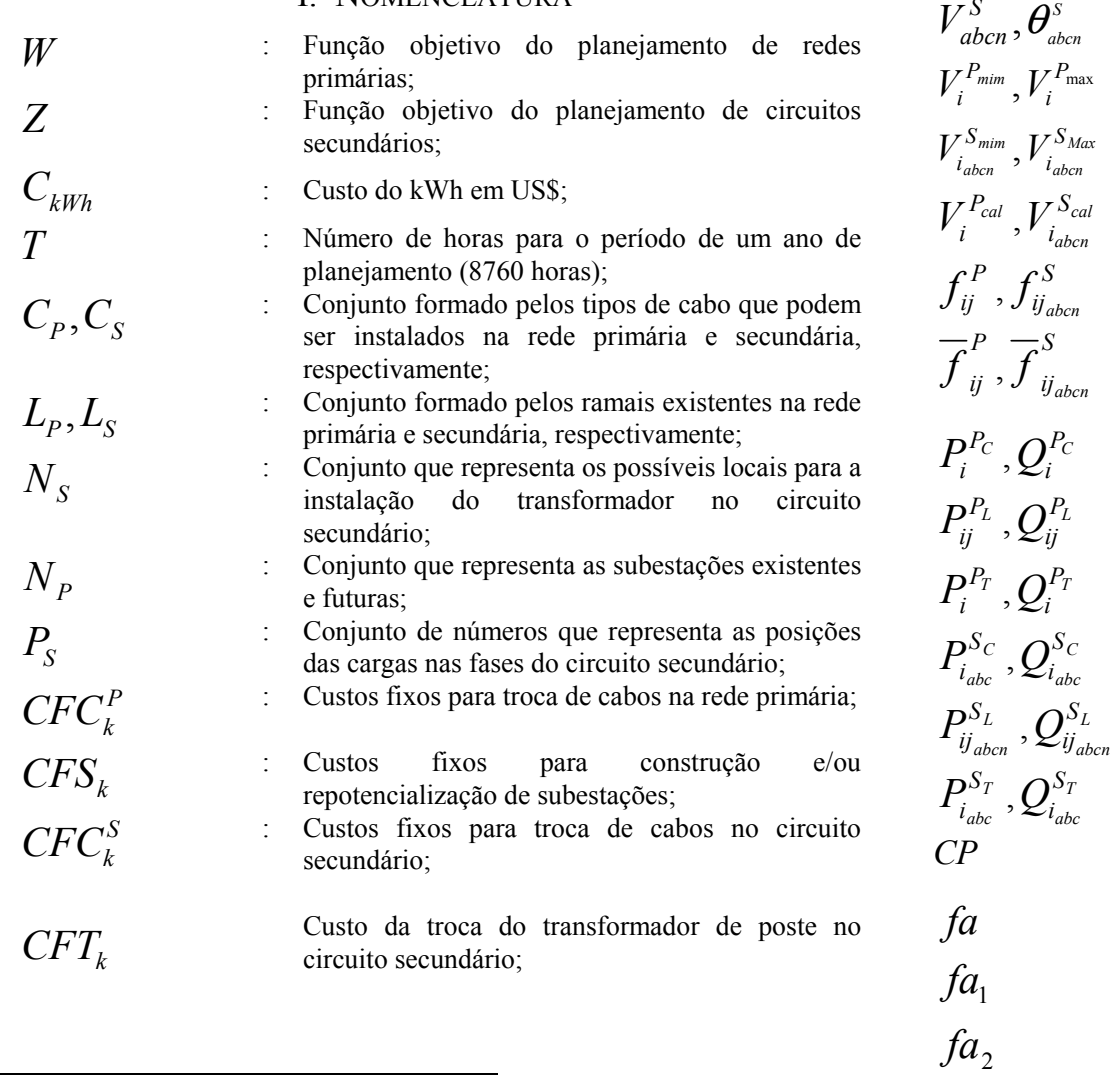

Antônio. M. Cossi é engenheiro do Departamento de Planejamento de Sistemas de Distribuição de Energia Elétrica das Empresas Rede Energia, em Presidente Prudente-SP, amcossi@gmail.com.

José. R. S. Mantovani trabalha como professor adjunto da Universidade Estadual Paulista, Campos de Ilha Solteira-SP, mant@dee.feis.unesp.br.

$$
\begin{aligned}
& C F B_{k} \\
& y_{k, i j}^{P}, y_{k, i j}^{S} \\
& \text { ti } \\
& \text { li } \\
& f i \\
& R_{k, i j}^{P}, R_{i j_{a b c n}}^{S} \\
& I_{i j}^{P}, I_{i j_{a b c n}}^{S} \\
& P_{D}^{P}, Q_{D}^{P} \\
& V^{P}, \theta^{P} \\
& P_{D_{a b c n}}^{S}, Q_{D_{a b c n}}^{S} \\
& V_{a b c n}^{S}, \boldsymbol{\theta}_{a b c n}^{S} \\
& V_{i}^{P_{\operatorname{mim}}}, V_{i}^{P_{\max }} \\
& V_{i_{a b c n}}^{S_{\operatorname{mim}}}, V_{i_{a b c n}}^{S_{M a x}} \\
& V_{i}^{P_{c a l}}, V_{i_{a b c n}}^{S_{c a l}} \\
& f_{i j}^{P}, f_{i j a b c n}^{S} \\
& \bar{f}_{i j}^{P}, \bar{f}_{i j_{a b c n}}^{S} \\
& P_{i}^{P_{C}}, Q_{i}^{P_{C}} \\
& P_{i j}^{P_{L}}, Q_{i j}^{P_{L}} \\
& P_{i}^{P_{T}}, Q_{i}^{P_{T}} \\
& P_{i_{a b c}}^{S_{C}}, Q_{i_{a b c}}^{S_{C}} \\
& P_{i j_{a b c n}}^{S_{L}}, Q_{i j_{a b c n}}^{S_{L}} \\
& P_{i_{a b c}}^{S_{T}}, Q_{i_{a b c}}^{S_{T}} \\
& \text { CP } \\
& \text { fa } \\
& f a_{2}
\end{aligned}
$$

Custo do balanceamento das cargas nas fases do circuito secundário;

Variável de decisão para alterar (1) ou não (0) a bitola do cabo no ramo $i-j$ da rede primária e do circuito secundário, respectivamente;

: Variável de decisão para construção (1) ou não (0) de subestações na rede primária;

: Variável de decisão para mudança (1) ou não (0) do transformador de posição no circuito secundário;

: Variável de decisão para o balanceamento (1) ou não (0) das cargas no circuito secundário;

Resistência dos condutores no ramo $i-j$ a ser construído ou recondutorado na rede primária e no circuito secundário, respectivamente;

Corrente no ramo $i-j$ da rede primária e do circuito secundário, respectivamente;

Demandas de potências ativa e reativa na rede primária;

: Magnitude e ângulo de tensão nodal na rede primária;

: Demanda de potências ativa e reativa no circuito secundário;

Magnitude e ângulo de tensão nodal no circuito secundário;

Limite da magnitude de tensão na barra $i$ (mínima e máxima) da rede primária;

: Limite da magnitude de tensão na barra $i$ (mínima e máxima) do circuito secundário;

: Tensão calculada na barra $i$ da rede primária e do circuito secundário, respectivamente;

: Fluxos calculados nos ramos $i-j$ da rede primária e do circuito secundário, respectivamente;

: Máximos fluxos permitidos nos ramos $i-j$ da rede primária e do circuito secundário, respectivamente;

: Potências ativa e reativa consumidas na barra $i$ da rede primária;

: Perdas ativa e reativa no ramo $i-j$ da rede primária;

: Capacidade da subestação - Potências ativa e reativa;

: Potências ativa e reativa consumidas na barra $i$ do circuito secundário;

: Perdas ativa e reativa no ramo $i-j$ do circuito secundário;

: Capacidade dos transformadores do circuito secundário - Potências ativa e reativa;

: Conjunto de propostas de interligações entre os sistemas de $M T$ e de $B T$;

: Função custo total do planejamento integrado;

: Função de adaptação do planejamento de redes primárias;

: Função de adaptação do planejamento de circuitos secundários.

\section{INTRODUÇÃO}

problema do planejamento da expansão dos sistemas de distribuição de energia elétrica é de grande importância 
para as empresas distribuidoras devido o crescimento do consumo de energia elétrica e a necessidade de atender aos consumidores com qualidade de fornecimento de energia, altos índices de confiabilidade e custos competitivos. Assim, altos custos de investimentos e de operação, somados ao fato que o sistema de distribuição é o que se conecta diretamente com os consumidores, ressaltam a importância do planejamento da expansão destes sistemas. Ações desenvolvidas a partir de técnicas de otimização e heurísticas adotadas no processo de planejamento, podem acarretar ganhos substanciais nos custos de expansão e operação. Isto justifica o desenvolvimento de programas computacionais como ferramenta de análise e planejamento que promovam a confiabilidade e a qualidade do fornecimento de energia elétrica de maneira econômica, levando-se em consideração o montante dos investimentos a serem aplicados nos projetos de planejamento de redes de distribuição.

O sistema de distribuição é subdividido em dois subsistemas, primário (média tensão) e secundário (baixa tensão) [1], [15]. Devido o elevado grau de dificuldade imposto pela natureza combinatória do problema de planejamento destes dois subsistemas, a maioria das ferramentas computacionais para análise e planejamento trata o problema do planejamento de redes primárias separadamente do planejamento dos circuitos secundários de distribuição, reduzindo assim a dimensão do espaço de busca do problema. Poucos trabalhos são encontrados na literatura que tratam do problema do planejamento de sistemas de distribuição contemplando de forma integrada os subsistemas primário e secundário. Tal abordagem é de grande importância já que os resultados do planejamento de um subsistema influencia nos resultados do outro, tendo como conseqüência um planejamento completo, ainda mais eficiente, com a redução dos custos totais e das perdas. Neste contexto, encontra-se o trabalho desenvolvido em [8], que trata do problema de planejamento da distribuição de forma hierarquizada dos objetivos, contemplando simultaneamente aos dois subsistemas, sendo o subsistema primário resolvido em um nível superior e o secundário no nível inferior.

Vários métodos têm sido empregados no estudo do planejamento ótimo de sistemas de distribuição, sistemas primários, secundários ou planejamento integrado (redes de $M T$ e BT): técnicas analíticas - branch-and-bound [14], Modelos de Transportes e decomposição de Benders [8], [10], Métodos Heurísticos [12] e atualmente as Meta-Heurísticas Algoritmos Evolutivos [1], [7], Simulated Annealing e Busca Tabu [2], [5].

Neste trabalho os problemas de planejamento de redes primárias e circuitos secundários de distribuição são formulado como problemas de programação não linear inteiro mistos (PNLIM) [1], [2], [10], [13]. Para integrar o planejamento dos sistemas de $M T$ e de $B T$ propõe-se uma técnica heurística composta por um conjunto de regras que são encontradas na prática ao se fazer as conexões entre as redes de $M T$ e $B T$. O planejamento integrado de sistemas de distribuição tem por objetivos obter resultados de forma conjunta, tanto na expansão quanto na operação do sistema como um todo, melhorando assim a qualidade técnica do serviço de fornecimento de energia elétrica a um custo total reduzido. Para isso, propõe-se um conjunto de alternativas que são analisadas interativa e hierarquicamente para interligar da melhor forma possível os subsistemas primário e secundário, $\mathrm{e}$ obter o planejamento otimizado do ponto de vista técnico e econômico dos sistemas de $M T$ e de $B T$.

Para testar a eficiência do modelo de planejamento integrado, apresentam-se resultados de testes realizados em sistema real composto por uma rede primária e circuitos secundários de distribuição de energia elétrica [16].

\section{MODElo MATEMÁTICO}

\section{A. Função Objetivo do Planejamento de Redes Primárias}

O planejamento da expansão de sistemas primário de distribuição de energia elétrica pode ser formulado como um problema de otimização não linear inteiro misto $(P N L I M)$ de grande porte, da seguinte forma:

$$
\begin{aligned}
& \text { MIin } W=\sum_{k \in C_{P}} \cdot \sum_{i j \in L_{P}}\left[C F C_{k}^{P}+\left(C_{k W h}\right) \cdot(T) \cdot\left(R_{k, i j}^{P}\right) \cdot\left(\left|I_{i j}^{P}\right|^{2}\right)\right] \cdot y_{k, i j}^{P} \\
& +\sum_{k \in N_{P}}\left(C F S_{k}\right) \cdot t i+\sum_{i j \in L_{P}}\left(C_{k W h}\right) \cdot(T) \cdot\left(R_{i j}^{P}\right) \cdot\left(\left|I_{i j}^{P}\right|^{2}\right) \cdot\left(1-\sum_{k \in C_{P}} y_{k, i j}^{P}\right)
\end{aligned}
$$

A equação (1) envolve os custos em investimentos na rede (construção de novas subestações e alimentadores e troca de cabos), além dos custos operacionais (perdas elétricas).

\section{B. Função Objetivo do Planejameto de Circuitos Secundários}

O planejamento de circuitos secundários de distribuição também pode ser formulado como um modelo de programação não linear inteiro misto (PNLIM), escrito matematicamente da seguinte maneira:

$$
\begin{aligned}
& \operatorname{Min} Z= \sum_{i j \in L_{S}}\left(C_{k W h}\right) \cdot(T) \cdot\left(R_{i j_{a b c n}}^{S}\right) \cdot\left(\left|I_{i j_{a b c n}}^{S}\right|^{2}\right) \cdot\left(1-\sum_{k \in C_{S}} y_{k, i j}^{S}\right) \\
&+\sum_{k \in C_{S}} \cdot \sum_{i j \in L_{S}}\left[C F C_{k}^{S}+\left(C_{k W h}\right) \cdot(T) \cdot\left(R_{k, i j a b c n}^{S}\right) \cdot\left(\left|I_{i j_{a b c n}}^{S}\right|^{2}\right)\right] \cdot y_{k, i j}^{S} \\
&+\sum_{k \in N_{S}}\left(C F T_{k}\right) \cdot l i+\sum_{k \in P_{S}}\left(C F B_{k}\right) \cdot f i
\end{aligned}
$$

$\mathrm{Na}$ equação (2) minimizam-se os custos em investimentos no circuito (balanceamento de fases, instalação e/ou troca de condutores, repotencialização e posicionamento adequado de transformadores no circuito) e os custos operacionais (perdas técnicas).

\section{Restrições}

As restrições físicas, operacionais e de qualidade do serviço de fornecimento de energia elétrica consideradas nos modelos de planejamento de redes primárias e circuitos secundários, podem ser representadas matematicamente como: 


$$
\begin{aligned}
& g\left(P_{D}^{P}, Q_{D}^{P}, V^{P}, \theta^{P}\right)=0 \\
& g\left(P_{D_{a b c n}}^{S}, Q_{D_{a b c n}}^{S}, V_{a b c n}^{S}, \theta_{a b c n}^{s}\right)=0 \\
& V_{i}^{P_{\operatorname{mim}}} \leq V_{i}^{P_{c a l}} \leq V_{i}^{P_{\max }} \\
& V_{i_{a b c n}}^{S_{m i m}} \leq V_{i_{a b c n}}^{S_{c a l}} \leq V_{i_{a b c n}}^{S_{M a x}} \\
& \left|f_{i j}^{P}\right| \leq \bar{f}_{i j}^{P} \\
& \left|f_{i j_{a b c n}}^{S}\right| \leq \bar{f}_{i j_{a b c n}}^{S} \\
& \sum_{i \in N B_{P}} P_{i}^{P_{C}}+\sum_{i j \in L_{P}} P_{i j}^{P_{L}} \leq \sum_{i \in N t_{P}} P_{i}^{P_{T}} \\
& \sum_{i \in N B_{P}} Q_{i}^{P_{C}}+\sum_{i j \in L_{P}} Q_{i j}^{P_{L}} \leq \sum_{i \in N t_{P}} Q_{i}^{P_{T}} \\
& \sum_{i \in N B_{S}} P_{i_{a b c}}^{S_{C}}+\sum_{i j \in L_{S}} P_{i j_{a b c n}}^{S_{L}} \leq \sum_{i \in N t_{S}} P_{i_{a b c}}^{S_{T}} \\
& \sum_{i \in N B_{S}} Q_{i_{a b c}}^{S_{C}}+\sum_{i j \in L_{S}} Q_{i_{j a b c n}}^{S_{L}} \leq \sum_{i \in N t_{S}} Q_{i_{a b c}}^{S_{T}} \\
& \sum_{k \in C_{P}} y_{k, i j}^{P} \leq 1 \quad, \forall i j \in L_{P} \\
& \sum_{k \in C_{S}} y_{k, i j}^{S} \leq 1 \quad, \forall i j \in L_{S}
\end{aligned}
$$

Através de (3) e (4) verifica-se diretamente o estado da rede primária e dos circuitos secundários, respectivamente, em que as leis de Kirchhoff (lei das correntes e das tensões) asseguram o balanço de demanda para cada nó do circuito, fazendo com que o somatório do fluxo líquido de potência seja nulo. As equações (5) e (6) impõem que o perfil de tensão da rede primária e dos circuitos secundários seja mantido dentro de limites preestabelecidos, evitando grandes variações de tensões. As equações (7) e (8) asseguram o atendimento do limite da capacidade de operação dos alimentadores da rede primária e circuitos secundários (existentes ou futuros), durante o período de planejamento. Nas equações (9) e (10) assegura-se que a capacidade total das subestações seja suficiente para atender à demanda durante o período de planejamento. As equações (11) e (12) garantem que a capacidade total dos transformadores seja suficiente para atender a demanda e as perdas técnicas nos circuitos secundários durante o período de planejamento. As equações (13) e (14) estabelecem que apenas um tipo de cabo pode ser escolhido para ser instalado ou trocado num possível recondutoramento dos ramais da rede primária e dos circuitos secundários, respectivamente.

\section{TÉCNICA DE SOLUÇÃO}

A técnica de solução adotada para o planejamento integrado consiste de um procedimento iterativo em que no primeiro nível, para cada uma das propostas de interligação entre as redes de $M T$ e $B T$, é resolvido o planejamento do circuito de $M T$ através de um algoritmo de busca tabu dedicado. No segundo nível, considerando-se os resultados obtidos no primeiro nível é efetuado o planejamento do circuito de $B T$. As propostas de interligação entre os circuitos de $M T$ e de $B T$ consistem de um conjunto de possibilidades para integrar o circuito de $M T$ com os circuitos de $B T$. Estas possibilidades são estabelecidas de forma heurística baseadas nas condições geográficas, físicas e elétricas existentes entre o circuito de $M T$ e os circuitos de $B T$.

\section{A. Planejamento de Redes Primárias}

Para resolver o problema do planejamento de redes primárias de distribuição de energia elétrica é utilizado um algoritmo TS [2], [3], [4], [6]. O algoritmo TS foi desenvolvido para explorar as características específicas do problema de planejamento, obter confiabilidade dos resultados e eficiência computacional. Para mapear as possíveis soluções para o problema é utilizada uma codificação em base decimal inteira que determina as propostas de conexão dos alimentadores e o tipo de cabo a ser instalado e/ou trocado, obedecendo à restrição de radialidade imposta ao problema. Este sistema de codificação está ilustrado na Fig. 1.

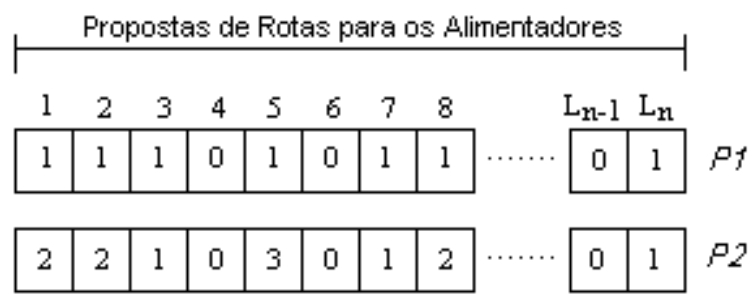

Figura 1. Sistema de Codificação.

Neste esquema de codificação tem-se:

i. $\quad$ P1: Propostas de conexão dos alimentadores no sistema (1 - cabo alocado no ramo $L$ da rede, para $L=1, \ldots, L_{n} ; 0$ ramo sem cabo alocado);

ii. P2: Tipo de cabo proposto para cada alimentador conectado ao sistema.

No esquema de codificação da Fig. 1 e na transição entre configurações vizinhas, deve ser obedecida a restrição de radialidade.

$\mathrm{O}$ algoritmo $T S$ parte de uma configuração inicial com o conjunto de vizinhos pré-determinados através da seguinte heurística construtiva:

O algoritmo Busca Tabu Reativo parte de uma configuração inicial com o conjunto de vizinhos conhecidos. Para gerar a configuração semente inicial é utilizada uma heurística construtiva que consiste em criar conexões entre as subestações existentes e futuras e as novas cargas que devem ser energizadas, através da construção de novos alimentadores e ramais. $\mathrm{Na}$ construção dos novos ramais e alimentadores, 
subestações novas e repotencialização das existentes, considera-se a capacidade de potência disponível das subestações, a radialidade do sistema, as propostas preestabelecidas para construção de ramais e alimentadores para energizar as novas cargas que devem ser ligadas no sistema. Esta heurística consiste dos seguintes passos:

i. Escolher um nó de carga $j$ para ser energizado;

ii. Escolher uma subestação $\mathrm{Si}$ existente com capacidade de fornecimento de energia disponível e que ainda não foi analisada para energizar a carga $j$. Fazer $S \leftarrow S_{i}$ e ir ao passo iii;

iii. Verificar a capacidade de operação da subestação $S$ com a adição do nó $j$ :

a. Se a capacidade de operação da subestação $S$ não for ultrapassada, ligar a carga $j$ através de um ramal no alimentador desta subestação mais próximo da carga $j$. A bitola do condutor deste ramal é especificada como sendo o cabo de menor bitola para ser especificado no planejamento da rede de distribuição . Ir para o passo $i v$;

b. Caso contrário, retirar o nó $j$ da subestação $S$ e ir para o passo $v$.

iv. Verificar a radialidade do sistema devido à adição do nó $j$ à subestação $S$ :

a. Se o sistema for radial, ir ao passo vi;

b. Caso contrário, retirar o nó $j$ da subestação $S i$ e voltar ao passo $i$.

v. Verificar se existem subestações $S_{k}$ com capacidade de potência disponível. Se existe subestação com capacidade de potência disponível, escolher a subestação $S_{k}$ que possui alimentadores mais próximos do ponto de carga $j$. Fazer $S \leftarrow S_{k}$, voltar ao passo iii. Caso contrário, escolher dentre as subestações propostas para serem construídas ou repotencializadas, a subestação $S_{k}$ de menor custo. Fazer $S \leftarrow S_{k}$ e retornar ao passo iii.

vi. Verificar se todos os nós de carga estão energizados:

a. Se existir nós com carga desenergizados, ir ao passo $i$

b. Caso contrário, ir para o passo vii.

vii. Construir o vetor codificação da topologia obtida para o sistema de distribuição inicial.

A estrutura de vizinhança é dividida em duas partes. A primeira, referente à criação de rotas dos alimentadores, é baseada na técnica de troca de ramos [12] realizada em duas zonas: intrazona - realiza a troca de ramos conectados a uma mesma subestação e interzona - realiza a troca de ramos conectados em subestações diferentes. Esta técnica consiste em adicionar ramos que não fazem parte da configuração corrente formando uma nova topologia (árvore) como proposta de solução para o problema de planejamento. Para manter a radialidade do sistema, um outro ramo (aresta) é retirado desta configuração proposta, diferentemente daquele que foi adicionado. Assim, um vizinho de uma configuração corrente é qualquer sistema que seja diferente em um ramo desta configuração, conservando a sua radialidade. A segunda estrutura de vizinhança refere-se à escolha da bitola do cabo a ser substituído (recondutorado) ou alocado no sistema. Essa escolha é feita através da avaliação das condições de fluxo para os condutores existentes na configuração sob estudo e para aqueles pertencentes ao conjunto de condutores candidatos a serem alocados.

Cada proposta de planejamento obtida para cada vizinhança do TS é avaliada através de uma função de adaptação $f a l$ que considera a função objetivo (1) e violações do conjunto de restrições do PNLIM, que são consideradas através de penalizações. $O$ estado operacional da rede para avaliar o conjunto de restrições é obtido através do processamento de um fluxo de potência monofásico [9]. Este algoritmo de fluxo de potência monofásico é adequado para o planejamento de redes primárias por ser rápido e eficiente e também porque as redes primárias podem ser consideradas durante a fase de planejamento equilibradas ou com muito pouco desequilíbrio. No procedimento de planejamento das redes primárias através da metodologia proposta há a necessidade de resolver uma grande quantidade de problemas de fluxo de potência. Na Fig. 2 apresenta-se um diagrama de blocos da metodologia proposta para o planejamento do sistema de $M T$.

\section{B. Planejamento de Circuitos Secundários}

A técnica de solução proposta para o planejamento de circuitos secundários de distribuição é um algoritmo evolutivo $(A E)$, desenvolvido e adaptado para solução deste problema específico [1]. Uma codificação em base decimal inteira é utilizada para mapear as possíveis soluções do problema, a qual é dividida em três subconjuntos: o primeiro refere-se ao balanceamento de cargas nas fases $a b c$ do circuito, o segundo determina o tipo de cabo a ser instalado em cada ramal e o terceiro localiza o ponto ideal para a instalação do transformador na rede. A população inicial é gerada de forma aleatória e é capaz de evoluir com a aplicação dos operadores genéticos (seleção, recombinação e mutação) propiciando o surgimento de configurações que levem às possíveis soluções ótimas do problema. Para a seleção das melhores configurações utilizou-se a técnica do torneio com elitismo. O processo de recombinação é de um único ponto para cada um dos diferentes subconjuntos de variáveis (balanceamento de cargas, bitola dos condutores e posicionamento do transformador na rede), mapeados pela estrutura do cromossomo. Neste caso, a parcela de uma configuração é trocada com a parcela correspondente da outra configuração do mesmo par selecionado de acordo com a taxa de recombinação preestabelecida. Para efetuar a mutação, para cada um dos setores de variáveis que compõem o cromossomo, procede-se da seguinte maneira: para o balanceamento de cargas nas fases do circuito faz-se uma rotação nas cargas das fases $a, b$ e $c$ (trocar cargas das fases); para a bitola dos cabos é escolhido um com bitola acima ou abaixo da existente, sendo esta escolha de forma aleatória; e para o posicionamento do transformador é alterada sua 
posição para um poste a jusante ou a montante do mesmo, sendo esta escolha também aleatória.

Para cada indivíduo da população que representa uma proposta de planejamento do circuito secundário é calculada uma função de adaptação $f a_{2}$. Dada à natureza não linear do problema, esta função é modelada considerando a função objetivo (2) e as penalizações das restrições violadas, cujos parâmetros são obtidos através do processamento de um fluxo de potência trifásico a quatro fios [4]. Este modelo de fluxo de potência é necessário para o planejamento de circuitos secundários porque é nestes circuitos que estão os maiores desequilíbrios de cargas nas fases do sistema de distribuição de energia elétrica. Na Fig. 3 apresenta-se um diagrama de blocos da metodologia proposta para o planejamento do sistema de $M T$.

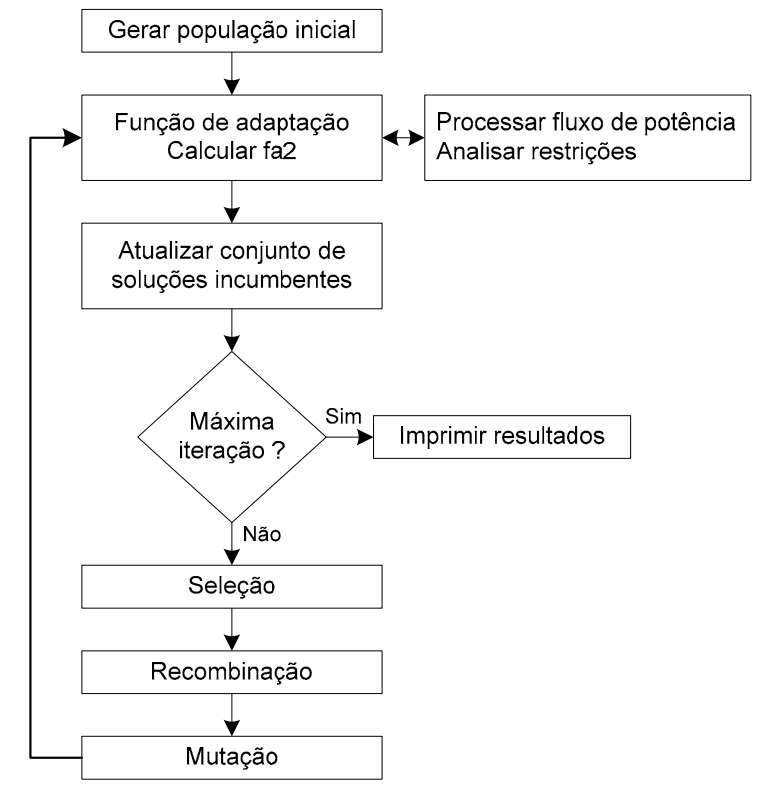

Figura 2. Diagrama de blocos metodologia proposta para o planejamento de sistemas de BT utilizando algoritmo evolutivo.

\section{Planejamento Integrado}

No planejamento integrado, procura-se verificar a influência entre os resultados do planejamento dos subsistemas primário e secundário, de forma a minimizar os custos totais de investimentos e operação do sistema de distribuição como um todo. Desta forma no planejamento integrado se consideram as alternativas de planejamento de cada subsistema, primário e secundário, além das restrições dos modelos de planejamento de cada um dos subsistemas.

No desenvolvimento do algoritmo para efetuar o planejamento integrado, devem ser previstas algumas condições que contemplem a estrutura física, geográfica e topológica das redes existentes e as necessidades de expansão do sistema.

A integração entre os sistemas primários e circuitos secundários é realizada através dos alimentadores primários que deverão possuir os possíveis pontos para a instalação dos transformadores abaixadores e derivação dos circuitos de $B T$. Para tal, deve-se conhecer o roteamento dos alimentadores primários e os possíveis pontos e ramais de interconexão com os circuitos secundários. A seleção desses pontos de derivação dos circuitos secundários pode ser considerada então como uma expansão do subsistema primário.

Neste trabalho considera-se que o planejamento integrado é efetuado da seguinte forma:

1) Resolve-se o planejamento da rede primária considerando uma proposta de interconexão com o circuito secundário, de acordo com uma lista de propostas, e com base no crescimento de carga do sistema (expansão ou construção de um novo circuito secundário);

2) Resolve-se o planejamento do circuito secundário de acordo com a proposta de integração adotada no planejamento primário;

3) Com base nos resultados de ambos os planejamentos, a avaliação do custo global do planejamento para cada proposta de integração, através de uma função de adaptação $f a$, definida como:

$$
f a=f a_{1}+f a_{2}
$$

A melhor solução é aquela que contempla a integração com um menor custo de planejamento, mas de forma que obedeça aos critérios técnicos de operação da rede. Na Fig. 3 ilustra-se o diagrama de blocos do modelo de planejamento integrado entre os sistemas de $M T$ e de $B T$.

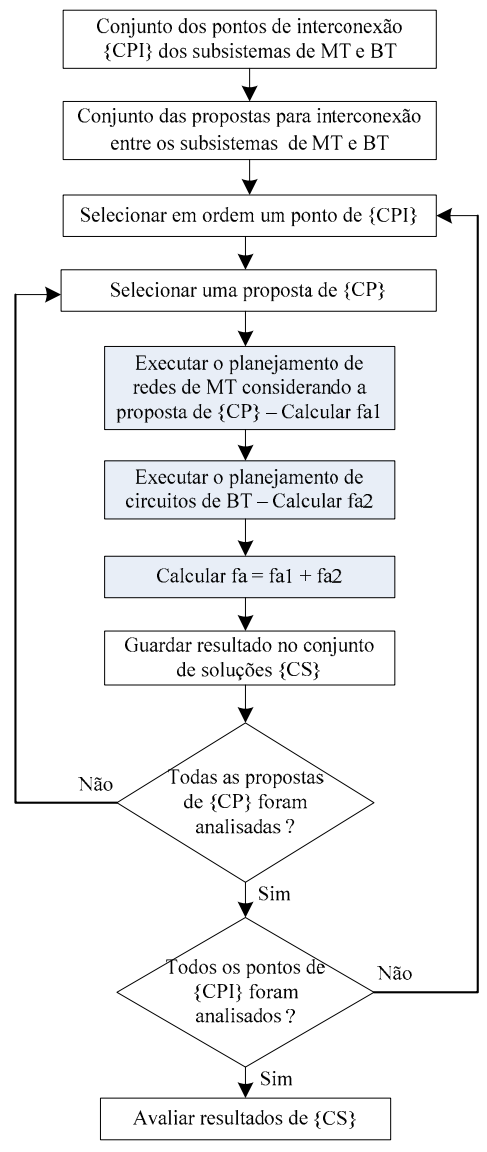

Figura 3. Diagrama ilustrativo da metodologia proposta para planejamento integrado de sistemas de distribuição de MT e de AT utilizando Tabu Search. 
A heurística para promover este planejamento integrado, considerando os aspectos técnicos e econômicos dos subsistemas de $M T$ e $B T$ é composta dos seguintes passos:

$i$. Conhecer o roteamento dos alimentadores primários $\mathrm{e}$ secundários do sistema com base nos índices de crescimento de cargas e fatores sócio-econômicos e ambientais;

ii. Promover o planejamento integrado (buscar pelas melhores soluções para um planejamento integrado de redes primárias e circuitos secundários):

a. Com base em uma lista de propostas para a interconexão entre os subsistemas primário e secundário, adotar uma proposta de acordo com a ordem das mesmas para realizar o planejamento primário (previsão de cargas);

$b$. Promover o planejamento primário, considerando o crescimento de carga do sistema (expansão ou construção de um novo circuito secundário). Neste caso, cada proposta de interconexão entre estes subsistemas consiste em executar as seguintes ações:

1. Construir novos alimentadores primários conectando o circuito secundário diretamente à subestação, e/ou;

2. Conectar o circuito secundário através da expansão de alimentadores primários existentes;

3. Avaliar a capacidade da subestação: caso a subestação esteja operando em sua máxima capacidade, avaliar a possibilidade de repotencialização da mesma. Caso não seja possível, avaliar a viabilidade de construção de uma nova.

c. Para cada proposta de interconexão entre os subsistemas primário e secundário adotado no planejamento primário, efetuar o planejamento do circuito secundário e analisar possíveis ações como:

1. Balanceamento de cargas nas fases;

2. Recondutoramento dos ramais secundários;

3. Troca do transformador abaixador: caso o transformador esteja operando em sua máxima capacidade, promover a troca do transformador por outro de maior potência;

4. Localização do transformador: analisar a mudança do transformador abaixador de posição de acordo com a proposta adotada no planejamento primário para a interconexão entre os subsistemas primário e secundário;

5. Remanejamento de cargas: analisar a possibilidade de remanejamento de cargas de um circuito secundário para outro circuito adjacente, caso seja necessário.

d. Verificar o critério de parada:
1. Se todas as propostas de interconexão entre os subsistemas primário e secundário foram analisadas, ir para o passo iii.

2. Caso contrário, guardar os resultados e voltar ao item a do passo ii.

iii. Avaliar o custo total do planejamento integrado para cada proposta de interconexão entre os subsistemas primário e secundário analisadas no passo ii, considerando as ações de planejamento adotado em cada um destes subsistemas;

$i v$. Imprimir resultados.

No planejamento integrado, devido às várias opções a serem executadas para promover a interconexão entre os subsistemas primário e secundário, não se obtém apenas uma única solução, mas um conjunto de soluções em que a escolha da solução mais conveniente a ser adotada depende das condições de projetos que sejam mais viáveis tecnicamente.

\section{RESUltados}

Para testar o sistema computacional implementado em linguagem de programação FORTRAN a partir da metodologia proposta, foram realizados testes em um sistema primário, trifásico equilibrado a três fios real em expansão constituído por 84 barras, 62 ramais existentes e 97 propostos para atender novos consumidores, incluindo as opções de roteamento para a integração dos sistemas primário $\mathrm{e}$ secundário [16]. O circuito secundário utilizado, também constitui um sistema trifásico a quatro fios, real com 72 barras e 71 ramais [16]. Na Fig. 4, os alimentadores ilustrados na cor vermelha indicam os possíveis pontos adotados para a integração entre a rede primária e o circuito secundário sob análise. Na Fig. 5 está ilustrada a configuração da solução do que apresenta o menor custo global para o planejamento integrado. Para esta configuração, o menor valor da magnitude de tensão nas barras é de 0.963 pu. Na Tabela I estão os custos de planejamento da rede primária para cada opção de ligação para integrar a rede primária com o circuito secundário e o perfil de tensão máximo e mínimo da configuração obtida como solução do planejamento integrado. Na Tabela II estão os custos para as melhorias propostas na rede secundária, considerando-se as opções de alocação do transformador nas barras de integração com a rede primária, sendo a barra 1 (85 da rede primária) onde o transformador abaixador se encontra instalado. Na Tabela III estão os custos totais para cada proposta de planejamento integrado entre os subsistemas primário e secundário. 


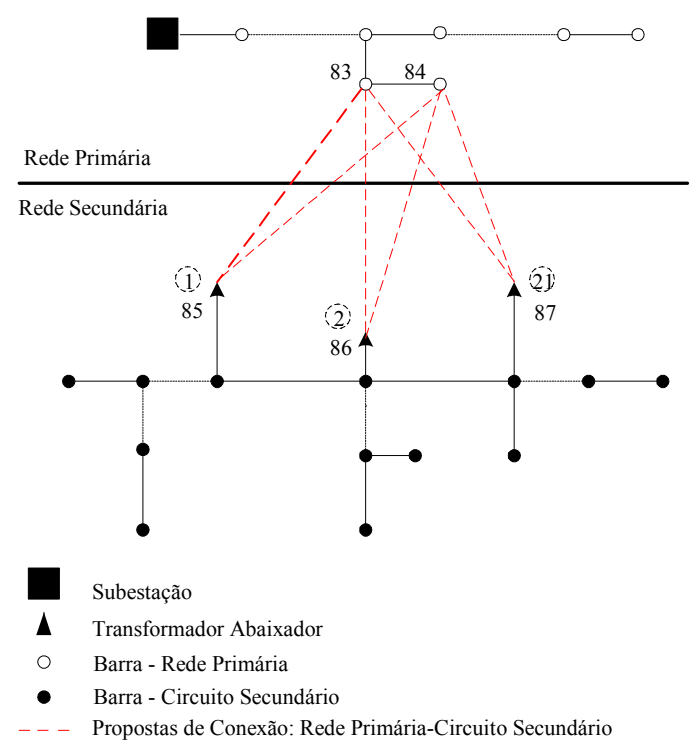

Figura 4. Possíveis roteamentos para integração entre a rede primária e o circuito secundário.

TABELA I

CUSTOS DO PLANEJAMENTO PRIMÁRIO PARA CADA OPÇ̃̃o DE INTEGRAÇ̃̃o ENTRE A REDE PRIMÁRIA E SECUNDÁRIA

\begin{tabular}{|c|c|c|c|c|}
\hline Barras & Distância & \multicolumn{3}{|c|}{ Custos $\boldsymbol{f a}_{\boldsymbol{1}}$ (US\$) } \\
\cline { 3 - 5 } Integração & $(\mathbf{k m})$ & Perdas & Cabos & Total \\
\hline $83-85$ & 0,0372 & 7568.68 & 7624.08 & 15192.77 \\
\hline $83-86$ & 0,0489 & 7609.58 & 7947.18 & 15556.77 \\
\hline $83-87$ & 0,0672 & 7705.26 & 8048.80 & 15754.07 \\
\hline $84-85$ & 0,0984 & 7870.12 & 8478.73 & 16348.85 \\
\hline $84-86$ & 0,0724 & 7758.08 & 8252.91 & 16011.00 \\
\hline $84-87$ & 0,0260 & 7529.01 & 7624.08 & 15153.10 \\
\hline
\end{tabular}

TABELA II

MELHORIAS NA REDE SECUNDÁRIA DE ACORDO COM AS OPÇÕES DE PLANEJAMENTO INTEGRADO

\begin{tabular}{|l|c|c|c|c|}
\hline \multirow{2}{*}{$\begin{array}{c}\text { Custos } \boldsymbol{f a}_{2} \\
\text { (US\$) }\end{array}$} & \multirow{2}{*}{$\begin{array}{c}\text { Sistema } \\
\text { atual }\end{array}$} & \multicolumn{3}{|c|}{ Configurações Propostas } \\
\cline { 3 - 5 } & $\begin{array}{c}\text { Trafo } \\
\text { barra 1(85) }\end{array}$ & $\begin{array}{c}\text { Trafo } \\
\text { barra 2(86) }\end{array}$ & $\begin{array}{c}\text { Trafo } \\
\text { barra 21(87) }\end{array}$ \\
\hline Operação & 389.22 & 202.87 & 223.49 & 235.97 \\
\hline $\begin{array}{l}\text { Balanceamento } \\
\text { de cargas }\end{array}$ & 0,00 & 37.55 & 37.55 & 42.25 \\
\hline $\begin{array}{l}\text { Realocação do } \\
\text { transformador }\end{array}$ & 0,00 & 0,00 & 37.55 & 37.55 \\
\hline Troca de cabos & 0,00 & 59.43 & 59.43 & 69.55 \\
\hline Total & 389.22 & 299.86 & 358.04 & 358.34 \\
\hline Tensão (V) & Vmin & 0.967 & 0,96 & 0.953 \\
\cline { 2 - 5 } & Vmax & 1.00 & 1.00 & 1.00 \\
\hline Custo/Benefício & & 0.52 & 0.81 & 0.97 \\
\hline
\end{tabular}

TABELA III

Custo Total do Planejamento Integrado dos Subsistemas PrimáRIO E SECUNDÁRIO

\begin{tabular}{|c|c|c|c|}
\hline \multirow{2}{*}{$\begin{array}{c}\text { Barras de } \\
\text { Integração }\end{array}$} & \multicolumn{3}{|c|}{ Custos (US\$) } \\
\cline { 2 - 4 } & $\boldsymbol{f a}_{\boldsymbol{1}}$ & $\boldsymbol{f a}_{\boldsymbol{2}}$ & $\boldsymbol{f a}$ \\
\hline $83-85(1)$ & 15192.77 & 299.86 & 15492.63 \\
\hline $83-86(2)$ & 15556.77 & 358.04 & 15914.81 \\
\hline $83-87(21)$ & 15754.07 & 358.34 & 16112.41 \\
\hline $84-85(1)$ & 16348.85 & 299.86 & 16648.71 \\
\hline $84-86(2)$ & 16011.00 & 358.04 & 16369.04 \\
\hline $84-87(21)$ & 15153.10 & 358.34 & 15511.44 \\
\hline
\end{tabular}

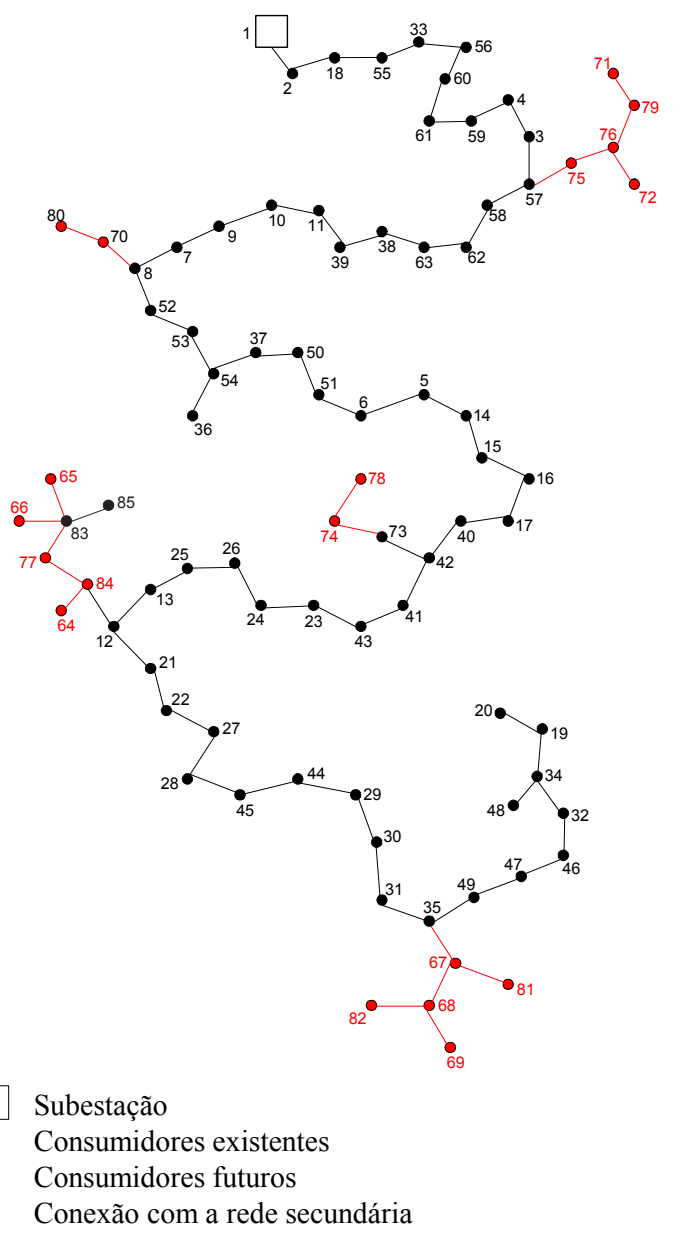

Figura 5. Configuração solução da rede primária após o planejamento integrado.

\section{A. Análise dos Resultados}

Analisando os resultados do planejamento da rede primária na Tabela I, conclui-se que a melhor opção para integração, do ponto de vista econômico é através das barras 84 e 87 (barra 21 do circuito secundário) conforme ilustra a Fig. 4, pois esta é a opção que possui a menor distância entre as redes, configurando-se como os melhores pontos adjacentes para a ligação do circuito secundário. No circuito secundário a melhor opção para integrá-lo com a rede primária é através da barra 1 (barra 85 da rede primária), pois nos outros casos os investimentos realizados não compensam os benefícios, como mostra a relação custo/benefício para cada opção de integração com a rede primária na Tabela II. Fazendo-se uma análise do custo total, obtido para cada proposta de integração dos subsistemas, conforme mostra a Tabela III, a melhor opção de integração é através das barras 83 e 85 (barra 01 do circuito secundário), conforme ilustrado na Fig. 4. Neste caso, além de não precisar mudar o transformador de posição no circuito secundário, evitando assim o aumento de custos, o sistema opera com níveis de perdas mais baixos e custos reduzidos quando comparado com as outras opções de integração. No entanto, dependendo das condições de projeto pode-se adotar outra solução dentre as fornecidas pelo programa de planejamento. Na Tabela III verificam-se 
diferentes propostas com custos atraentes para interconexão entre os subsistemas primário e secundário que podem ser adotadas dependendo das condições de projeto para a rede de distribuição.

\section{CONCLUSÕES}

Os resultados dos testes realizados mostram que a metodologia proposta permite obter soluções otimizadas para o problema de planejamento de redes primárias e de circuitos secundários. A qualidade destas soluções depende do conjunto de opções possíveis para integrar a rede primária e os circuitos secundários de distribuição,que é construído com base na experiência do planejador e nas condições geográficas, elétricas e físicas do sistema sob estudo. Para solução dos modelos de planejamento das redes de $M T$ e de $B T$ podem ser utilizadas qualquer uma das metaheurísticas encontradas na literatura [3].

\section{AGRADECIMENTOS}

Os autores agradecem as empresas do Grupo Rede, a Fundação de Ensino, Pesquisa e Extensão de Ilha Solteira FEPISA e o Conselho Nacional de Desenvolvimento Científico e Tecnológico - CNPq (Processo 301060/2006-1), pelo apoio financeiro durante o desenvolvimento deste projeto de pesquisa.

\section{REFERÊNCIAS}

[1] A. M. Cossi, R. Romero, and J. R. S. Mantovani, "Planning of secondary distribution circuits through evolutionary algorithms," IEEE Transactions on Power Delivery, vol. 20, no. 1, pp. .205-213, 2005.

[2] A. M. Cossi, R. Romero, A. P. Feltrin e J. R. S. Mantovani, "Planejamento de redes primárias de distribuição através de um modelo de programação não linear inteiro misto e busca tabu reativa," XXXVII Simpósio Brasileiro de Pesquisa Operacional, pp. 1418-1429, 2005.

[3] C. R Reeves, Modern HeuristicTtechniques for Combinational Problems, Ingland: Library of congress Catalogin-in-publication Data, 1993, p. 320.

[4] R. M. Ciric, A. Padilha-Feltrin, and L. F. Ochoa, "Power flow in fourwire distribution networks-general approach," IEEE Transactions on Power Systems, vol. 18, no. 4, pp. 1283-1290, 2003.

[5] F. A. B. Lucero, "Planejamento de sistemas de distribuição de energia elétrica utilizando algoritmo tabu search," Dissertação de mestrado, Faculdade de Engenharia, Unesp de Ilha Solteira, 2003.

[6] F. Glover, and G. A. Kochenberger, Handbook of Metaheuristics, Library of congress, 2003, p. 570.

[7] I. J. Ramírez-Rosado, and J. L. Bernal-Agustín, "Genetic algorithms applied to the design of large power distribution systems," IEEE Transactions on Power Systems, vol. 13, no. 2, pp. 693-703, 1998.

[8] M. C. da Silva, "Planejamento à longo prazo em sistemas de distribuição de energia elétrica," Tese de doutorado, Faculdade de Engenharia Elétrica e de Computação, Universidade Estadual de Campinas, 1990.

[9] M. E. Baran, and F. F. Wu, "Optimal capacitor placement on radial distribution system," IEEE Transactions on Power Systems, vol. 4, no. 1, pp. 725-734, 1989.

[10] N. Kagan, "Electrical distribution system planning using multi objective and fuzzy mathematical programming," Ph.D thesis, University of London, 1992.

[11] Procedimentos de Distribuição de Energia Elétrica no Sistema Elétrico Nacional - PRODIST, Agência Nacional de Energia Elétrica - ANEEL, 2006.

[12] S. K. Goswami, "Distribution system planning using branch exchange technique," IEEE Transactions on Power Systems, vol. 12, no. 2, pp, 718-723, 1997.
[13] S. R. Kato, and L. C. Leung, "Power distribution planning: a review of models and issues," IEEE Transactions on Power Systems, vol. 12, no. 3, pp. 1151-1158, 1997.

[14] D. I. Sun, D. R. Farris, P. J. Cote, R. R. Shoults, and M. S. Chen, "Optimal distribution substation and primary feeder via the fixed charge network formulation," IEEE Transactions on Power Apparatus and Systems, vol. 101, no. 3, pp. 602-609, 1982.

[15] T. Gönnen, Electric Power Distribution System Engineering, Ed. New York: McGraw-Hill, 1986, p. 752.

[16] Unesp-Feis, "Electrical energy eystems planning laboratory homepage - testsystems," Disponível: www.dee.feis.unesp.br/lapsee/

Antônio Marcos Cossi adquiriu o curso de graduação, título de Mestre e de Doutor em Engenharia Elétrica pela Unesp/Ilha Solteira - SP, Brazil, em 2001, 2003 e 2008, respectivamente.

Atualmente é engenheiro do setor de planejamento de sistemas de distribuição de energia elétrica das empresas Rede Energia, em Presidente Prudente-SP, Brazil.

José Roberto Sanches Mantovani adquiriu o curso de graduação em 1981 pela UNESP/Ilha Solteira-SP, título de Mestre e de Doutor em Engenharia Elétrica pela Universidade Estadual de Campinas/Unicamp-SP, Brazil, em 1987 e 1995, respectivamente.

Atualmente é professor adjunto do departamento de engenharia elétrica da Unesp/Ilha Solteira - SP, Brazil. 